DOI:

10.1038/nrg1886

URLs

\section{MOUSE GENETICS}

\section{No Mickey-} Mouse \section{phenomics}

The current emphasis on large-scale biology means that we require contemporary technologies to be high throughput or at least scalable. For genomics and genotyping, this goal is largely being achieved, but phenotyping analysis has notably lagged behind. So the new method for rapid, high-resolution phenotypic assessment of mouse embryos developed by John Johnson and Mark Hansen and colleagues should meet with much interest.

Laborious, histology-based phenotypic analysis has been improved by the introduction of magnetic resonance microscopy (MRM), but this approach requires highly specialized equipment and is therefore prohibitively expensive to most researchers. Johnson and Hansen et al. now report a new method that is based on $\mathrm{X}$-ray microscopic computed tomography (microCT). In this approach, a virtual histological representation of an embryo, which had been stained in $1 \%$ osmium tetroxide solution, is obtained at up to $8-\mu \mathrm{m}$ resolution.

The resolution of microCT exceeds that of routine MRM scans. Importantly, scanning takes less time, is less expensive and several embryos can be scanned at once. To maximize the high-throughput aspect of this approach, the authors advocate scans at $25-27 \mu \mathrm{m}$ (which is comparable to most MRM methods). With a small animal CT scanner, these only take 2 hours for up to 120 embryos. A higher resolution scan can always be done on a subset of the sample.

The authors tested their approach by characterizing Pax3:Fkhr transgenic mouse embryos, which are known to have complex neural tube defects. To this end they used a scanner that is similar to commercially available small animal in vivo scanners, which are frequently operated in regional core facilities. The new approach provided resolution that was as good as conventional histology of paraffin-embedded samples; moreover, it provided a clearer overall picture of the defects in three dimensions.

Among the drawbacks of this technique is its dependence on osmium tetroxide staining - because permeability of embryos decreases as the epidermis develops, the approach is most suited to embryos in midgestation. Embryos that are older than embryonic day 13.5 need to be treated with protease or have the epidermis removed manually.

As the authors point out, highthroughput phenotyping will also require automated or semi-automated computer-based analysis of the data. Given the recent advances in microimaging and high-resolution microCT instrumentation, and the use of existing and new electrondense stains, who knows, true phenomics might be coming soon to a laboratory near you.

\section{Magdalena Skipper}

ORIGINAL RESEARCH PAPER Johnson, J. T \& Hansen, M. S. et al. Virtual histology of transgenic mouse embryos for high-throughput phenotyping. PLoS Genet. 2, e61 (2006)

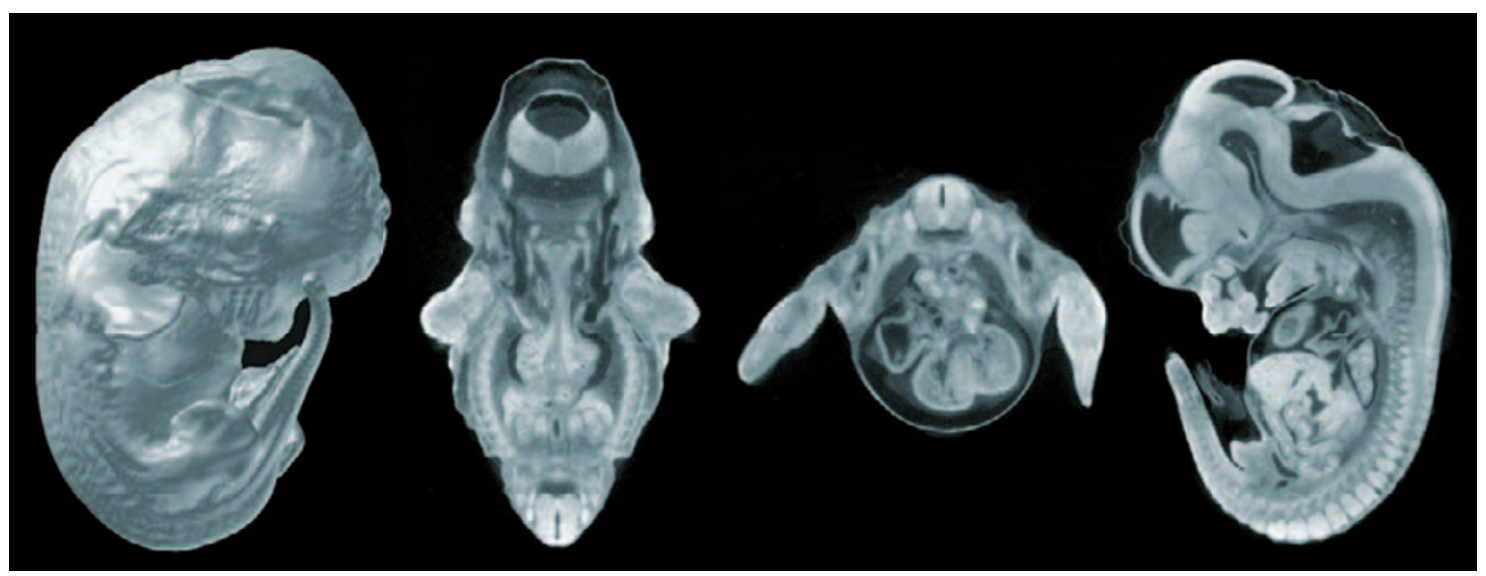

Image courtesy of Charles Keller, University of Texas Health Sciences Center at San Antonio, and John T. Johnson, Scientific Computing and Imaging Institute, University of Utah 